\title{
Effect of hospital ethical climate on the nurses' moral sensitivity
}

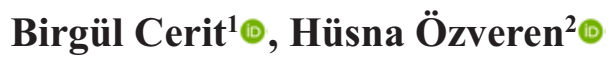 \\ ${ }^{1}$ Department of Nursing, Bolu Abant Izzet Baysal University, Bolu Health School, Bolu, Turkey \\ ${ }^{2}$ Department of Nursing, Kirlkkale University, Faculty of Health Sciences, Kırlkkale, Turkey
}

DOI: $10.18621 /$ eurj.423324

\begin{abstract}
Objectives: The study determines the effects of the hospitals ethical climate on the moral sensitivity of nurses. Methods: The study design is descriptive and correlational. The data was obtained from 99 nurses who voluntarily accepted to fully participate in the surveys conducted. The data was analysed using descriptive statistics and Pearson correlations.

Results: As a result of the study, the ethical climate perception of nurses and their moral sensitivity level was found to be above average. A positive, meaningful and medium relationship was found between the moral sensitivity level of nurses and ethical climate perceptions of nurses.

Conclusions: Our findings support that the ethical climate perception of nurses is an important factor in determining the moral sensitivity. Consequently, building an ethical climate in all the hospitals that provide health care will result in a high moral sensitivity of nurses when giving health care.
\end{abstract}

Keywords: ethical climate, hospital, moral sensitivity, nurses, nursing ethics

Received: May 14, 2018; Accepted: July 5, 2018; Published Online: September 17, 2018

$\mathrm{T}$ oday, hospitals strive to receive quality certification and high recognition. In this context, hospitals project the changes and innovations brought by science and technology on health care services, which can power them to carry on good and satisfactory treatment on the patients in their various field. The cost of quality and quantity of these services provided by hospitals are influenced by "moral sensitivity" and its crucial determinant "ethical climate" that reflect on the attitudes and decisions of health care professionals. Ethical climate which has various definitions in literature is described as the perception of atmosphere that increases ethical thoughts, mutual respect and trust in the organization and allows for questioning, discussions and expression of different views [1]. Ethical cli- mate is also defined as common general perceptions related to corporate organizational values, practices and operations. It also includes perceptions related to expected, supported and rewarded behaviors [2]. According to Victor and Cullen [3], ethical climate involves the characteristics that immensely influence decisions and experienced in organizations and this climate requires generating a common approach that seeks answers to the questions which arise when dealing with ethical problems and to accurate approaches that need to be used in terms of ethics.

Ethical climate is a concept related to the degree of implementing ethical norms [4]. Therefore, it is crucial for hospitals to create an ethical climate and ensure the existence of a work environment in which the

Address for correspondence: Birgül Cerit, RN, PhD., Assistant Professor, Bolu Abant Izzet Baysal University, Bolu Health School, Department of Nursing, 14030 Bolu, Turkey

E-mail: birgulcerit@yahoo.com.tr,Phone:+90 374 2534520,Fax:+90 3742534557 
employees adopt ethical principles so that hospitals can sustain quality health services, keep their existence and increase their organizational performances [5]. Creating a positive climate in hospitals and a robust organizational structure and operation born out of practicing ethical values may generate feelings of ownership in employees and decrease feelings of loneliness as well as having positive impact on productivity [6]. It may also have positive reflections on patient satisfaction with health care services.

Nurses who are the leading force in operating health care services are health professionals who are directly affected by the ethical climate patterns adopted by hospitals. Nurses experience despair and incapacity in displaying professional attitudes and behaviors in the ethical decision-making process regarding the ethical dilemmas they face while offering health care services. There are some environmental factors that hinder professional attitudes and generate negative feelings during the process. Although moral distress and ethical climate are the most important factors, there are many other elements such as lack of administrative support, limitations based on organizational procedures and policies and resource control $[7,8]$. In a study of nurses' ethical climate perception, McDaniel [9] found that nurses had fewer opportunities to participate in ethical negotiations in their institutions, were not adequately supported in practice, and had inconsistencies in policy and procedures in practice. In another study, it has been determined that nurses' having a more positive ethical climate perception of the institution they work at reduces the moral distress experienced [10]. It is crucial to create a positive work environment in organizations to ensure that employees can carry out their duties effectively because positive climate affects employees' morale, motivation and organizational commitments [6]. The ethical climate of an organization can act like a guide to solving ethical dilemmas experienced by employees and can also affect nursing practices and patient outcomes [10].

It is of important for nurses believe in the ethical climate atmosphere and the existence of an ethical climate approach in the hospital they work so that they can display ethical behaviors when carrying out their duties related to patient care. Ethical climate can also affect making ethically sound decisions while contributing to the development of moral sensitivity dur- ing the process. Moral sensitivity enhances individual competence used during ethical decision-making process [11]. In other words, moral sensitivity enables professionals to understand, interpret and appropriately respond to individuals who receive professional services [12]. According to Lovett, moral sensitivity is the skill of noticing moral problems [13] and prevents ethical dilemmas and conflicts in the process of ethical decision-making for patients. Therefore, the first step in displaying ethical behaviors in quality nurse care is to have moral sensitivity [14].

Moral sensitivity in professional practice develops through sensibility, sensitivity and intentness based communication of healthcare professionals in cases where patients experience difficulties and uncertainties as well as vulnerabilities. Moral sensitivity allows nurses and other health care professionals to morally respond to individuals who are receiving professional health care services, who are in pain and who are vulnerable [12]. Patients entrust their lives, bodies, health and their most vulnerable and private aspects to nurses who care for them. In this context, patient-nurse communication based on trust is of important. However, since nurses are relatively the stronger party in this professional relationship and they provide care for vulnerable groups, the moral aspects of care become clearer [15]. Professional care received by patients during their stay at the hospital brings ethical responsibilities for nurses. Since nurses' decisions and choices made by nurses include protecting the pride and supporting the comfort of patients who need care and treatment [16].

In this context, considering the moral aspect of nurse care and the ethical responsibilities it bestows on nurses, the importance of moral sensitivity and the ethical climate atmosphere for quality nurse care will be better comprehended. Policies followed by hospitals in the face of ethical problems and their reflections on organizational climate -i.e. ethical climate atmosphere generated by the hospital- may guide nurses' moral sensitivity and nursing practices and may affect the decisions made by nurses. It is important that nurses' decisions and actions are far from being intuitional, free from prejudices, and based on scientific, objective; and ethical principles. It is necessary to create an ethical climate to ensure professional nursing practices. In atmospheres where professionalism is ignored and ethical climate is not recognized, it is rather 
hard to have moral sensitivity in practices related to care, making decisions about the significance and priorities of ethical principles and values, and displaying behaviors with moral standards which are supported by professional attitudes [17]. Hence, creation of an ethical climate in the organization is indispensable for quality health care services more of it provides nurses with the opportunitiy to approach problems faced in patient care with moral sensitivity, effectively follow ethical decision-making process and work by conducting the requirements of professionalism.

\section{Objective}

The study aims to determine the effect of hospitals ethical climate perception on the nurses' moral sensitivity.

\section{Research Questions}

What is the nurses' perception of ethical climate in their work place?

1. What are the opinions of nurses about moral sensitivity level?

2. Is there a relationship betweennurses' perception of ethical climate in their work place and level of moral sensitivity?

\section{METHODS}

\section{Setting and Sample}

The study was descriptive and correlational in design. It was conducted in a state and university hospital in Kirıkkale, Turkey. The sample size was estimated using $\mathrm{G}$ power analysis for a power level of .90 , a significance level of .05 and medium effect size to enable the planned analyses. A sample size of 112 participant was calculated. The data was collected from 99 nurses who voluntarily accepted to participate and returned the surveys fully completed (88\% response rate).

\section{Ethical Considerations}

For the approval of the study, written permission was obtained from each hospital. Nurses were informed about the aims and methods of the study. They were informed about voluntarily participation and the right to withdraw from the study at any time. Permissions were granted for the use of MSQ and
HECS from Hale Tosun and Havva Öztürk respectively and from the Ethical Committee of Bolu Abant Izzet Baysal University (Protocol number: 2012/14).

\section{Data Collection}

The data were collected through face-to-face interviews. To obtain the descriptive qualities of the nurses, a personal information form was administered. The Turkish version of the Hospital Ethical Climate Survey was used to determine the ethical climate perception in hospitals, and The Turkish version of the Moral Sensitivity Questionnaire was used to identify the level of moral sensitivity.

\section{Instruments \\ Moral Sensitivity Questionnaire (MSQ)}

The Moral Sensitivity Questionnaire (MSQ) was originally developed by Lutzen [11] in 1994 to assess the moral sensitivity levels of the nurses. The MSQ was adapted into Turkish in 2003 by Tosun [18], and its validation and reliability were confirmed. The Turkish version of the MSQ is a 30-item selfadministered survey consisting of six sub-dimension (including autonomy: 7 items, benevolence: 4 items, meaning: 5 items, conflict: 3 items, rules: 4 items, and relation: 4 items) based on the 7-point Likert type scale. Three items are not included into any subdimension in this questionnaire. The scales ranged from 1 (completely disagree) to 7 (completely agree). A total score between $30-210$ is possible in this questionnaire. The higher scores indicate the higher levels of moral sensitivity of nurses $[18,19]$. With regard to reliability of the instrument, Tosun [18] reported a Cronbach's alpha value of 0.84 , Başak et al. [20] 0.80, Han et al. [21] 0.76, and Kim et al. [19] 0.85 . In this study, it was calculated as 0.88 .

\section{The Hospital Ethical Climate Survey (HECS)}

The Hospital Ethical Climate Survey (HECS) was originally developed by Olson in 1995 [22] to assess the ethical climate perception in their work place of nurses. The HECS was adapted into Turkish in 2003 by Bahçecik and Öztürk [23], and it was confirmed to be valid and reliable. The Turkish version of the HECS is a 24-item self-administered survey consisting of 5 sub-dimension (including patients: 4 items, managers: 6 items, hospital: 5 items, peers: 4 items, and 
physicians: 5 items) based on the 5-point Likert type scale, from 5 indicating "always true" to " 1 " indicating "not true" [23]. The total scale score of 24120 is possible. The higher scores indicate the positive perception of the hospital ethical climate of nurses. With regard to reliability of the instrument, Olson [22] reported a Cronbach's alpha value of 0.91, Bahçecik and Öztürk [23] 0.89, and Karagözoğlu et al. [2] 0.92. In this research, the Cronbach's alpha value was estimated as 0.95 .

\section{Statistical Analysis}

The SPSS, version 17.00 for Windows, was used for data entry and statistical analysis. Numbers and percentages were used in the analysis of sociodemographic data. In order to determine the ethical climate perception and views of nurses on moral sensitivity levels, mean and standard deviation were used. Pearson correlation analysis was used to determine the relationship between the ethical climate of the hospital and the moral sensitivity perception of nurses.

\section{RESULTS}

The characteristic features of the participating nurses were shown in Table 1 . Most $(\mathrm{n}=78 ; 78.8 \%)$ of the participating nurses was female, $48(48.5 \%)$ were between 27-35 years old, and $40(40.4 \%)$ had a bachelor's degree in nursing. Thirty-one $(31.3 \%)$ of the participants were working in medical ward, and 29 (29.3\%) had 6-10 years of nursing experience, and 44 (44.4\%) had 1-5 years of experience in their current wards, and $83(83.8 \%)$ had been working willingly in their current wards (Table 1).

Mean scores related to Nurses' perception of the ethical climate in their work place are shown in Table 2. In this study, the mean score of the nurses related to ethical climate perception was found to be $84.02 \pm$ 19.80 on a scale of 24-120, with range of 38-120. When the subscales were examined, it is also found that from the subscales of the HECS, the nurses got the highest point in peers $(15.46 \pm 3.69)$ and patients $(15.38 \pm 3.70)$ and the lowest points in hospitals $(15.78 \pm 4.93)$ (Table 2).

Table 1. General characteristic of participants $(n=99)$

\begin{tabular}{lccc}
\hline Characteristic & Category & $\mathbf{n}$ & \% \\
\hline Sex & Female & 78 & 78.8 \\
Age & Male & 21 & 21.2 \\
& $18-26$ & 21 & 21.2 \\
Educational background & $27-35$ & 48 & 48.5 \\
& $>36$ & 30 & 30.3 \\
Current place of employment & Senior high school & 22 & 22.2 \\
& Associate degree & 37 & 37.4 \\
& Bachelor & 40.4 & 31.3 \\
Experience as a nurse (years) & Medical ward & 31 & 27.3 \\
& Surgical ward & 27 & 27.3 \\
& Intensive care unit & 27 & 14.1 \\
& Other* & 14 & 7.1 \\
Experience at the ward (years) & $<1$ & 7 & 16.2 \\
& $1-5$ years & 16 & 29.3 \\
Work willingly at the ward & $6-10$ years & 29 & 23.2 \\
& $11-15$ years & 23 & 24.2 \\
& $>15$ & 24 & 32.3 \\
Other* & $<1$ & 32 & 44.4
\end{tabular}

Other* = emengency department, polyclinic, blood center 
Table 2. Mean scores of nurses obtained from hospital ethical climate survey $(n=99)$

\begin{tabular}{lcccc}
\hline Subscales of HECS & Item & Mean & SD & Total Score \\
\hline Patients & 4 & 15.38 & 3.70 & $4-20$ \\
Managers & 6 & 20.96 & 6.74 & $6-30$ \\
Hospital & 5 & 15.78 & 4.93 & $5-25$ \\
Peers & 4 & 15.46 & 3.69 & $4-20$ \\
Physicians & 5 & 16.42 & 4.67 & $5-25$ \\
Total Score & 24 & 84.02 & 19.80 & $24-120$ \\
\hline
\end{tabular}

$\mathrm{HECS}=$ hospital ethical climate survey, $\mathrm{SD}=$ standard deviation

The nurses MSQ average score was found to be $150.05 \pm 25.410$ on a scale of $30-210$, with range of 76-210. When the subscales were examined, it was also observed that from the subscales of the MSQ, the nurses got the highest point in autonomy (38.52 \pm $6.80)$ and relation $(21.83 \pm 4.55)$ and the lowest points in conflict (11.29 \pm 3.94$)$ (Table 3$)$.

The result of the correlation analysis made to determine whether there is a relationship between nurses' ethical climate perception and their moral sensitivity are given in Table 4 . The analysis results showed that there was a positive, meaningful and moderate relationship in between $(r=0.565)$. According to the correlation values, the highest relationship was found between the patients ethical climate and relation moral sensitivity subscales ( $\mathrm{r}=$ 0.603 ) and the lowest was found between peers and conflict $(r=0.208)$ subscales. According to the correlation values, a meaningful relationship was not found only between managers ethical climate and conflict moral sensitivity subscales $(\mathrm{r}=0.121)$ (Table $4)$.

Table 3. Mean scores of nurses in moral sensitivity questionnaire $(\mathrm{n}=99)$

\begin{tabular}{lcccc}
\hline Subscales of MSQ & Item & Mean & SD & Total Score \\
\hline Autonomy & 7 & 38.52 & 6.80 & $7-49$ \\
Benevolence & 4 & 20.05 & 4.11 & $4-28$ \\
Meaning & 5 & 26.44 & 6.27 & $5-35$ \\
Conflict & 3 & 11.29 & 3.94 & $3-21$ \\
Rules & 4 & 18.96 & 4.70 & $4-28$ \\
Relation & 4 & 21.83 & 4.55 & $4-28$ \\
Total Score & 30 & 150.05 & 25.41 & $30-210$ \\
\hline
\end{tabular}

$\mathrm{MSQ}=$ moral sensitivity questionnaire scores, $\mathrm{SD}=$ Standard deviation

Table 4. Correlations among hospital ethical climate survey scores and moral sensitivity questionnaire scores $(\mathrm{n}=$ 99)

\begin{tabular}{|c|c|c|c|c|c|c|}
\hline Subscales of Survey & Patients & Managers & Hospital & Peers & Physicians & $\begin{array}{r}\text { Total } \\
\text { HECS }\end{array}$ \\
\hline Autonomy & $0.574^{* *}$ & $0.437^{* *}$ & $0.522^{* *}$ & $0.484^{* *}$ & $0.560^{* *}$ & $0.609^{*}$ \\
\hline Benevolence & $0.420^{* *}$ & $0.217^{*}$ & $0.310^{* *}$ & $0.271^{* *}$ & $0.286^{* *}$ & $0.348^{*}$ \\
\hline Meaning & $0.552^{* *}$ & $0.365^{* *}$ & $0.404^{* *}$ & $0.455^{* *}$ & $0.434^{* *}$ & $0.516^{*}$ \\
\hline Conflict & $0.211^{*}$ & 0.121 & $0.233^{*}$ & $0.208^{*}$ & $0.288^{* *}$ & $0.246^{*}$ \\
\hline Rules & $0.372^{* *}$ & $0.338^{* *}$ & $0.404^{* *}$ & $0.380^{* *}$ & $0.539^{* *}$ & $0.483^{*}$ \\
\hline Relation & $0.603^{* *}$ & $0.377^{* *}$ & $0.407^{* *}$ & $0.509^{* *}$ & $0.375^{* *}$ & $0.526^{*}$ \\
\hline Total MSQ & $0.561^{* *}$ & $0.396^{* *}$ & $0.470^{* *}$ & $0.466^{* *}$ & $0.515^{* *}$ & $0.565^{*}$ \\
\hline
\end{tabular}

Data are shown as Pearson correlation coefficient (r). HECS $=$ hospital ethical climate survey, MSQ $=$ moral sensitivity questionnaire scores, ${ }^{*} p<0.05, * * p<0.01$ 


\section{DISCUSSION}

According to study results, nurses believed that ethical climate in their hospital was above average. Previous studies also pointed to similar findings [2, 10, 24-30]. Ethical climate in an organization can be evaluated by measuring employee perceptions related to organizational practices. Nurse perceptions regarding their work environment affect their approach to colleagues and the ethical problems they face as well as their attitudes towards solutions and their skills in making ethical decisions [1, 31]. Policies, procedures and practices of hospital in ethical matters may affect nurses' resolution to display attitudes and behaviors related to patient care [22, 24]. Ethical climate created in the hospital may cause moral distress in nurses $[10,24,27,32]$ and result in leave of employment [24, 31]. Therefore, ethical climate perceptions of health care professionals and especially of nurses who spend most time with patients and who provide continuous care is an important factor to ensure the provision of desired care and treatment [2]. Ethical climate perceptions of nurses are significant indicators to present nurse care based on moral sensitivity, ethical standards, trust and honesty. At the same time, ethical climate is the determinant factor in creating a robust organizational structure including the quality of organizational life at hospitals, employees' job satisfaction, motivation, commitment, patient and employee satisfaction, conflict prevention, identification of ethical problems and presentation of suitable ethical approaches and in offering quality health care services. In this respect, nurses believes that, they have an ethical climate which can be considered a positive satisfaction in terms of developing their moral sensitivity, taking care of the patients within the ethical approach and supporting the professional nursing roles.

This study found nurses' ethical sensitivity levels to be above average. Results of many studies in the literature are parallel to these findings [19, 20, 33-35]. Value problems experienced in relation to the changes and developments in health care services have resulted in new ethical problems and reflections of these problems on nurse care have made it compulsory for nurses to use ethical decision-making skills. Moral sensitivity has a significant place in using ethical decision-making skills. Moral sensitivity is required to identify ethical dilemmas and determine the appropriate values contained among alternative solutions [19]. However, moral sensitivity requires that the caregiver is sensible enough to comprehend verbal and non-verbal signs and behaviors to identify patient needs [36]. Moral sensitivity is also important to notice ethical dilemmas, produce solutions to problems and prevent conflicts [13, 19]. Decisions and choices made in the context of nurse care encumber nurses with immense responsibilities since they include protecting the pride of individuals in need of professional care and treatment, understanding their vulnerability, being aware of the moral outcomes of the decisions made for them and ensuring their comfort $[15,16,37]$. The findings in the study shows that nurses believing they have above average moral sensitivity may be important since it is an indicator that they will tend to display ethical appropriate behaviors.

The study identified a positive, significant and medium level relationship between nurses' hospital ethical climate perception and their moral sensitivity levels. Based on this finding, it can be claimed that nurses' positive ethical climate perceptions increase moral sensitivity. While ethical climate refers to individual organizational perceptions that affect attitudes and behaviors, it is also a reference to the course of action that will be displayed by employees [1]. Hence, policies, procedures and ethical climate perception related to hospitals are determinant factors for nurses to notice ethical dilemmas experienced in patient care and follows appropriate processes to solve these problems. Organizational obstacles and deadlocks in the process of solving ethical problems may result in less sensitivity in time [38]. Therefore, it is important for employees to believe in the existence of an ethical climate approach in the organization so that their moral sensitivity develops and conflicts and unethical behaviors can be prevented. Based on the results of this study, it can be stated that it is necessary to create ethical climates in hospitals to enhance nurses' moral sensitivity.

The study examined the relationships between hospital ethical climate sub dimension and moral sensitivity sub dimension and the highest level of relationship was found to be between patients and orientation sub dimension. According to this result, it can be expressed that; when nurses' ethical climate 
perceptions related to approaches towards patients increase, their interest in actions that can affect their relationships with patients will also rise. People are at the center of nursing practices. To offer high quality services that are humane, it is necessary to work in collaboration and provide nursing practices that do not ignore patient autonym and security. Nurses' positive ethical climate perceptions for their organization are crucial to present patient-centered approaches and sustain professionalism. Positive perceptions will affect nurse orientation and the standard of care provided. According to the result, it can be stated that belief in the existence of an organizational understanding that allows ethical thoughts ethical behaviors and free expression of ideas related to patient care; supports ethical decision-making skills based on trust and respect in the context of team work will positively contribute to orienting to the units where nurses work and the quality of patient care. The study shows that, the lowest degree of relationship between ethical climate sub dimensions and moral sensitivity sub dimensions was found between colleagues and conflict factors. The positive relationship between these two factors is an unexpected outcome when it is considered that communicating with colleagues is effective towards the systematic implementation of operations and conflict prevention. Literature review shows that the majority of nurses have not been trained in ethical issues [20,34-36] and they often ask the support of their colleagues to solve conflicts experienced during decision making process and to identify the correct action $[34,39,40]$. Since the majority of nurses generally do not receive training in ethics, it can be stated that decisions and actions based on professional seniority, inquisitive and personal judgments may be in effective. In these cases, suggestions provided by colleagues may contradict nurses' own sense of justice and value judgments and consequently the nurses may experience conflicts in approaching ethical problems and making ethical decisions. Appropriate approaches to ethical problems require that nurses are trained in ethical matters, make decisions about each action using their cognitive skills, use a systematic model as a guide and have moral sensitivity and awareness in addition to taking ethical theories and principles into consideration. Based on the results of the study, it can be stated that receiving support from colleagues in ethical issues may create conflicts and in order to eliminate conflicts, nurses should be provided with knowledge and skills in ethical matters and their moral sensitivity should be developed.

\section{CONCLUSION}

The perception of nurses related to their work place environment is important since it affects their approach to ethical problems and colleagues, their moral sensitivity, ethical decision skills and the quality of the care. As a result of the study, the ethical climate perception of the nurses and their moral sensitivity level was found to be above average. A positive, meaningful and medium relationship was found between the moral sensitivity levels and the ethical climate perceptions of the nurses. Our findings support that the ethical climate perception of the nurses is an important factor in determining the moral sensitivity. Therefore, that hospitals create an ethical climate will provide the nurses express themselves bravely in patient care, and plan and apply the patient care away from conflicts, coherent to patient rights, aiming to protect people's honour, with moral sensitivitiy and by using their ethical decision-making skills.

\section{Authors' Contributions}

$\mathrm{BC}$, and HÖ contributed to the conception, and design of this study. BC. performed the statistical analysis, interpretation of the data, and drafting the manuscript. H Ö. contributed to collection of the data. Both authors approved the final version of the manuscript. All authors are in agreement with the content of the manuscript.

\section{Conflict of interest}

The authors disclosed no conflict of interest during the preparation or publication of this manuscript.

\section{Financing}

The authors disclosed that they did not receive any grant during conduction or writing of this study.

\section{Acknowledgements}

This study was presented as an oral presentation at Turkish Bioethics Associations IX. Symposium in Turkey, 3-6 June 2016, Ankara. 
We would like to thank manegers of hospitals who permit to application of this study. We are also grateful to the participation of all nurses in this study.

\section{REFERENCES}

[1] Olson LL. Hospital nurses' perceptions of the ethical climate of their work setting. Image J Nurs Sch 1998;30:345-9.

[2] Karagözoğlu Ş, Özden D, Yıldırım G. [Intensive care nurses' perceptions of hospital ethical climate]. Hemşirelikte Araştırma Geliştirme Dergisi 2014;16:34-45. [Article in Turkish]

[3] Victor B, Cullen JB. The organizational bases of ethical work climates. Adm Sci Q 1988;33:101-25.

[4] Cullen JB, Parboteeah KP, Victor B. The effects of ethical climates on organizational commitment: a two-study analysis. J Bus Ethics 2003;46:127-41.

[5] Baykal Ü, Altuntaş S, Öztürk H, Sökmen S, İntepeler ŞS, Kantek F. [Ethical climate in the institution of nursing education]. İ.Ü.F.N. Hem. Derg 2012;20:26-34. [Article in Turkish]

[6] Eroğluer K, Yılmaz Ö. The effect of ethical leadership behavior on perceived organizational climate: mediating role of work loneliness. J Bus Res-Turk 2015;7:280-308.

[7] Crisham P. Measuring moral judgement in nursing dilemmas. Nurs Res 1981;30:104-10.

[8] Corley MC. Nurse moral distress: a proposed theory and research agenda. Nurs Ethics 2002;9:636-50.

[9] McDaniel C. Ethical environment: reports of practicing nurses. Nurs Clin North Am 1998;33:363-72.

[10] Pauly B, Varcoe C, Storch J, Newton L. Registered nurses' perceptions of moral distress and ethical climate. Nurs Ethics 2009;16:561-73.

[11] Lützen K, Cronqvist A, Magnusson A, Andersson L. Moral Stress: synthesis of a concept. Nurs Ethics 2003;10:312-22.

[12] Weaver K, Morse J, Mitcham C. Ethical sensitivity in professional practice: concept analysis. J Adv Nurs 2008;62:60718.

[13] Lovett BJ, Jordan AH. Levels of moralization: a new conception of moral sensitivity. J Moral Educ 2010;39:175-89.

[14] Ottekin-Demirbolat A, Aslan H. [An analysis of ethical sensitivity of primary and secondary schools in relation with students based on some variables (case of Sinop province)]. JESR 2014;4(1):187-202. [Article in Turkish]

[15] Dinç L. [The concept of caring and its' moral component]. Hacettepe University Faculty of Health Sciences Nursing Journal 2010;17:74-82. [Article in Turkish]

[16] Gastmans C. A fundamental ethical aproach to nursing: some proposals for ethics education. Nurs Ethics 2002;9:494-507.

[17] Şahin B, Dündar T. [Investigation of relationship among ethical climate and mobbing behaviors in health care sector]. Ankara Üniversitesi SBF Dergisi 2001;66(1):129-59. [Article in Turkish]

[18] Tosun H. Determining sensitivity of the nurses and the physicians against the ethical dilemmas which experienced at the health care practises. PhD Thesis, University of Istanbul, Istanbul, 2005.
[19] Kim YS, Kang SW, Ahn JA. Moral sensitivity relating to the application of the code of ethics. Nurs Ethics 2012;20: 470-8.

[20] Başak T, Uzun Ş, Arslan F. [Investigation of the moral sensibility of intensive care nurses]. Gülhane Med J 2010;52:7681. [Article in Turkish]

[21] Han SS, Kim J, Kim YS, Ahn S. Validation of a Korean version of the Moral Sensitivity Questionnaire. Nurs Ethics 2010;17:99-105.

[22] Olson L. Ethical climate in health care organizations. Int Nurs Rev 1995;42:85-90.

[23] Bahçecik N, Öztürk H. Hospital ethical climate survey in Turkey. JONAS Healthc Law Ethics Regul 2003;5: 94-9.

[24] Hwang JI, Park HA. Nurses' perception of ethical climate, medical error experience and intent-to-leave. Nurs Ethics 2014;21:28-42.

[25] Ghorbani AA, Hesamzadeh A, Khademloo M, Khalili S, Hesamzadeh S, Berger V. Public and private hospital nurses' perceptions of the ethical climate in their work settings, Sari City, 2011. Nurs Midwifery Stud 2014;3:1-7.

[26] Khalesi N, Arabloo J, Khosravizadeh O, Taghizadeh S, Heyrani A, Abbasali E. Psychometric properties of the Persian version of the "Hospital Ethical Climate Survey". J Med Ethics Hist Med 2014;7:1-7.

[27] Silen M, Kjellström, Christensson L, Sidenvall B, Svantesson M. What actions promote a positive ethical climate? A critical incident study of nurses' perceptions. Nurs Ethics 2012;19:501-12.

[28] Fazljoo E, Borhani F, Abbaszadeh A, Razban F. The ralitionship betwen nurses' perceptions of moral distress and ethical climate in Shahid Sadoughi University of Medical Sciences of Yazd. J Med Ethics Hist Med 2014;7:80-90.

[29] Allari R, Moghli FA. Moral distress among Jordian critical care nurse and their perception of hospital ethical climate. JNSR 2013;3: 144-53.

[30] Ulrich C, O'Donnell P, Taylor C, Farrar A, Danis M, Grady C. Ethical climate, ethics stress, and the job satisfaction of nurses and social workers in the United States. Soc Sci Med 2007;65:1708-19.

[31] Hart SE. Hospital ethical climates and registered nurses'turnover intentions. J Nurs Scholarsh 2005;37:173-7.

[32] Corley MC, Minick P, Elswick RK, Jacobs M. Nurse moral distress and ethical work environment. Nurs Ethics 2005;12:38190.

[33] Öztürk H, Hintistan S, Kasım S, Candaş B. [Ethical sensitivity of physicians and nurses in intensive care units]. Yoğun Bakım Hemşireliği Dergisi 2009;13:77-84. [Article in Turkish]

[34] Aksu T, Akyol A. [Investigation of the moral sensibility of nurses in İzmir]. Türkiye Klinikleri J Med Ethics 2011;19:16-24. [Article in Turkish]

[35] Dikmen Y. [An observation on the moral sensibility of intensive care nurses]. Cumhuriyet Nurs J 2013;2:1-7. [Article in Turkish]

[36] Schluter J, Winch S, Holzhauser K, Henderson A. Nurses' moral sensitivity and hospital ethical climate: a literature review. Nurs Ethics 2008;15:304-21.

[37] Lützén K, Blom T, Ewalds-Kvist B, Winch S. Moral stress, 
moral climate and moral sensitivity among psychiatric professionals. Nurs Ethics 2010;17:213-24.

[38] Epstein EG, Hamric AB. Moral distres, moral residue, and the crescendo effect. J Clin Ethics 2009;20:330-42.

[39] Grundstem-Amado R. Differences in ethical decision- making processes among nurses and doctors. J Adv Nurs 1992; 17:129-37.

[40] Jaeger SM. Teaching health care ethics: the importance of moral sensitivity for moral reasoning. Nurs Philos 2001;2:13142. 